\title{
Embeddings of cubic Halin graphs: Genus distributions*
}

\author{
Jonathan L. Gross \\ Columbia University, Department of Computer Science NY 10027 USA, New York, USA
}

Received 27 June 2011, accepted 8 April 2012, published online 1 June 2012

\begin{abstract}
We derive an $O\left(n^{2}\right)$-time algorithm for calculating the genus distribution of a given 3-regular Halin graph $G$; that is, we calculate the sequence of numbers $g_{0}(G), g_{1}(G)$, $g_{2}(G), \ldots$ on the respective orientable surfaces $S_{0}, S_{1}, S_{2}, \ldots$ Key topological features are a quadrangular decomposition of plane Halin graphs and a new recombinant-strands reassembly process that fits pieces together three-at-a-vertex. Key algorithmic features are reassembly along a post-order traversal, with just-in-time dynamic assignment of roots for quadrangular pieces encountered along the tour.
\end{abstract}

Keywords: genus distribution, Halin graph, partitioned genus distribution, gram embedding, outerplanar graph, topological graph theory.

Math. Subj. Class.: 05C10

\section{Introduction}

A Halin graph [20] is constructed from a plane tree $T$ with at least four vertices and no 2valent vertices by drawing a cycle thru the leaves of $T$ in the order they occur in a preorder traversal of $T$. Any wheel graph $W_{n}$ (for $n \geq 3$ ) is a Halin graph. Every Halin graph can be obtained by iterative splitting of the hub of a wheel and of some of the resulting vertices. Although some of the graphs obtained by splitting the hub of a wheel are non-planar, every planar graph so obtained is a Halin graph, since splitting a vertex of a tree yields a tree.

The outer cycle of a Halin graph is the cycle corresponding to the traversal of the leaves of the inscribed tree. [Since a Halin graph is 3-connected, its planar embedding is unique up to reversal of orientation, as per Whitney's theorem.] In the Halin graph of Figure 1, the outer cycle has length eight.

* This paper was presented as a keynote address at the 7th Slovenian International Conference on Graph Theory in June, 2011, at Lake Bled, Slovenia.

E-mail address: gross@cs.columbia.edu (Jonathan L. Gross) 


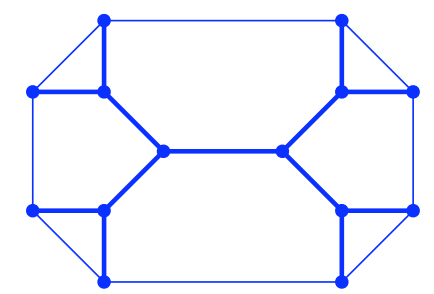

Figure 1: A Halin graph for a 14-vertex tree with 8 leaves.

\section{Genus distributions}

DEF. The genus distribution for graph $G$ is the sequence

$$
\gamma_{\text {dist }}(G): \quad g_{0}(G), \quad g_{1}(G), \quad g_{2}(G), \quad \cdots
$$

where $g_{i}(G)$ denotes the number of embeddings of $G$ in the orientable surface $S_{i}$ of genus $i$. In reckoning the number of embeddings of the graph $G$ in the surface $S$, we regard two embeddings $\iota: G \rightarrow S$ and $\iota^{\prime}: G \rightarrow S$ as the same if there is an extension of the identity automorphism $1_{G}: G \rightarrow G$ to an orientation-preserving autohomeomorphism $h: S \rightarrow S$ such that $\iota \circ h=\iota^{\prime}$ or, equivalently, if the two embeddings of $G$ correspond to the same rotation system (see [19]).

Calculating the genus distribution of a graph requires determining not only its minimum genus and its maximum genus, but also the number of embeddings of every possible genus. Table 1 gives the genus distributions of some familiar graphs, each of which is small enough that its genus distribution can be calculated by hand using ad hoc methods.

Table 1: Genus distributions of some familiar graphs.

\begin{tabular}{c|rrrrrl} 
graph $G$ & $g_{0}(G)$ & $g_{1}(G)$ & $g_{2}(G)$ & $g_{3}(G)$ & $g_{4}(G)$ & $\ldots$ \\
\hline$K_{4}$ & 2 & 14 & 0 & 0 & 0 & $\ldots$ \\
bouquet $B_{2}$ & 4 & 2 & 0 & 0 & 0 & $\ldots$ \\
dipole $D_{3}$ & 2 & 2 & 0 & 0 & 0 & $\ldots$ \\
$K_{3,3}$ & 0 & 40 & 24 & 0 & 0 & $\ldots$ \\
$K_{2} \times C_{3}$ & 2 & 38 & 24 & 0 & 0 & $\ldots$ \\
\hline
\end{tabular}

The study of genus distributions began with [16]. Some of the early papers, such as [9] and [18], were devoted to calculating genus distributions for all the graphs in a recursively constructible sequence. Other early papers, such as [33] and [7], were concerned with statistical properties of the distribution. A solution to a genus distribution calculation problem can be either a formula or a polynomial-time algorithm.

Lists of some previous papers on genus distributions have appeared in [10], [15], [17], and [29]. Papers published (or written) subsequently include the following: [4], [6], [5], [11], [12], [22], [23], [30], and [31].

\section{Graph amalgamations and bar-amalgamations}

In general, amalgamating two graphs means identifying a subgraph in one of them to an isomorphic subgraph in the other. Figures 2 and 3 illustrate vertex-amalgamation and 
edge-amalgamation, respectively, which are the two simplest kinds of amalgamation of two graphs.

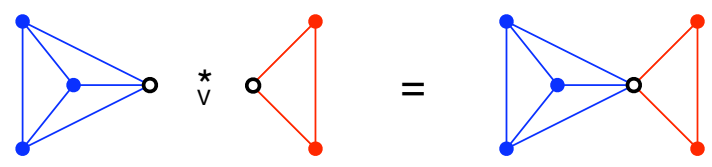

Figure 2: Vertex-amalgamation of two graphs.
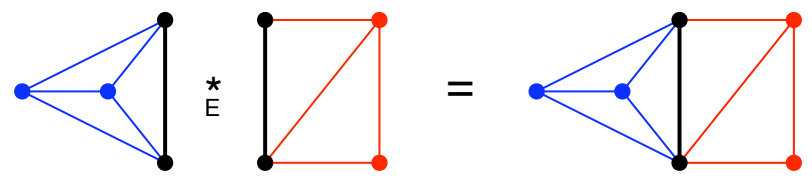

Figure 3: Edge-amalgamation of two graphs.

A bar-amalgamation of two (disjoint) graphs $G$ and $H$ is obtained by joining a vertex $u$ of $G$ to a vertex $v$ of $H$ with a new edge. It is denoted here by $G \bar{*} H$. Figure 4 shows a bar-amalgamation.
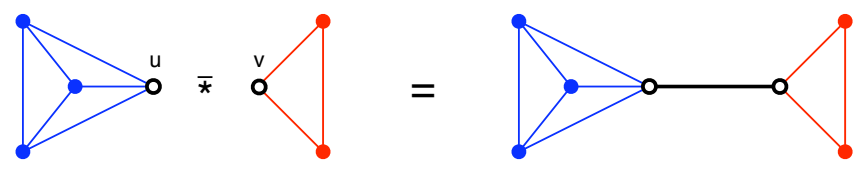

Figure 4: Bar-amalgamation of two graphs.

Proposition 1.1 ([16]). Let $G$ and $H$ be (disjoint) connected graphs, and let $u$ and $v$ be vertices of $G$ and $H$, respectively. Then

$$
\gamma_{\text {dist }}(G \bar{*} H)=\operatorname{deg}(u) \cdot \operatorname{deg}(v) \cdot \gamma_{\text {dist }}(G) \circ \gamma_{\text {dist }}(H)
$$

where $\circ$ means the operation of convolution on two sequences.

\section{Seeking a useful algorithm}

The objective herein is to derive a quadratic-time algorithm for calculating the genus distribution of any 3-regular Halin graph. The focus is not merely on proving the existence of such an algorithm, but on developing an algorithm that can by executed (albeit tediously) by hand for graphs with 10-20 vertices and rather quickly by a computer for graphs with a significantly larger number of vertices.

The terminology used here is consistent with [19] and [1]. For additional background (with some terminological differences), see [3], [28], or [37]. All of our graph embeddings here are cellular and orientable. A graph is taken to be connected, unless one can infer otherwise from the immediate context. Here we refer to a face-boundary walk as an $\boldsymbol{f b}$ walk.

Thanks to Imran Khan for creating the genus-distribution computer program (based on the Heffter-Edmonds algorithm) used in the course of this research.

\section{Known results concerning genus distributions}

Although calculating the maximum genus $\gamma_{\max }(G)$ of a graph $G$ is possible in polynomial time [8], calculating the minimum genus $\gamma_{\min }(G)$ is NP-hard [36], and calculating the 
genus distribution $\gamma_{\text {dist }}(G)$ is clearly at least as hard as calculating the minimum genus. Accordingly, rather few genus distributions are known. A survey of genus distributions, including average genus, is given by [10].

The most familiar such kinds of ladder graphs whose genus distribution formulas are known are as follows:

closed-end ladders [9] (derived 1984) See Figure 5.

circular ladders and Möbius ladders [26] See Figure 6.

Ringel ladders [35] See Figure 7.

By systematic use of iterated amalgamations [29] of double-edge-rooted graphs, self-edgeamalgamations [30], and edge-addition surgery [11], the calculation of formulas for these ladder graphs has been substantially simplified. Moreover, these recently developed techniques have produced quadratic-time algorithms for various generalizations of ladders, in which arbitrary graphs of known partitioned genus distribution (see $\S 4$ ) lie between the rungs.

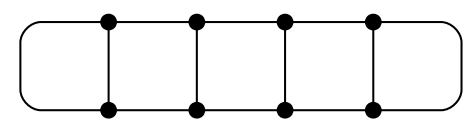

Figure 5: The closed-end ladder $L_{4}$.
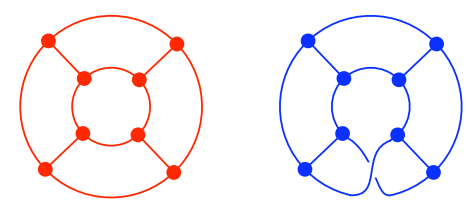

Figure 6: Circular ladder $C L_{4}$; and Möbius ladder $M L_{4}$.

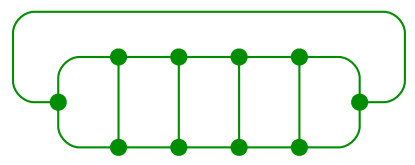

Figure 7: Ringel ladder $R L_{4}$.

A recent paper [13] presents a quadratic-time algorithm for the calculation of the genus distribution of any 3-regular outerplanar graph (see Figure 8). It uses a post-order traversal (see $\S 3$ ) and edge-amalgamations [29]. A subsequent paper [30] uses vertex-amalgamations [17] to derive a quadratic-time algorithm for 4-regular outerplanar graphs. Whereas outerplanar graphs are of tree-width 2, Halin graphs are of tree-width 3 (see [2]), which is intuitively a reason for anticipating the necessity for a more complicated analysis. Restrictions to 3-regularity or 4-regularity generally simplify the analysis of a genus distribution problem.

Some genus-distribution deriviations use a formula of Jackson [21] based on the theory of group representations. Bouquets, which are graphs with a single vertex and a number of self-loops (see Figure 10) were the first class to be so derived [18] . 


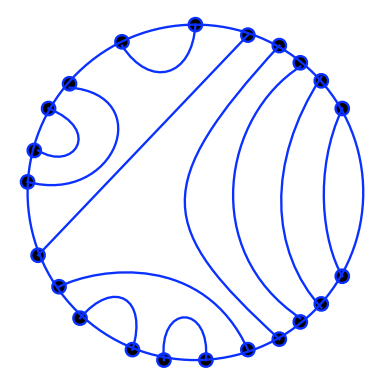

Figure 8: A 3-regular outerplanar graph.

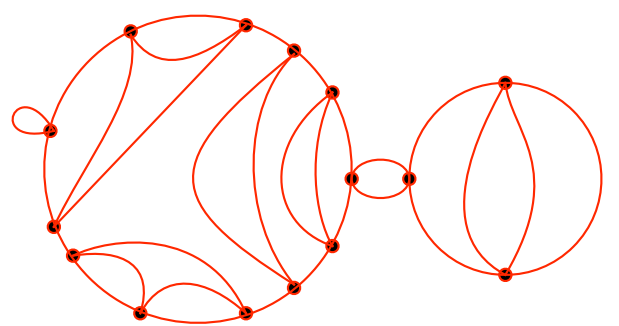

Figure 9: A 4-regular outerplanar graph.
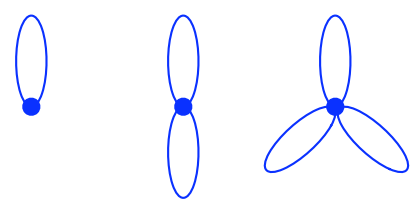

Figure 10: Bouquets $B_{1}, B_{2}$, and $B_{3}$.

Another such class is dipoles, which are graphs with two vertices and a number of edges joining them (see Figure 11). Their genus distributions are given by [32] and [24]. Yet another is fans, which are graphs obtained by joining a path graph to a single new vertex (see Figure 12). Their genus distributions were derived by [6].
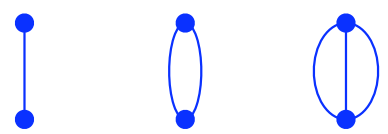

Figure 11: Dipoles $D_{1}, D_{2}$, and $D_{3}$.
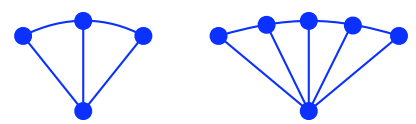

Figure 12: Fans $F_{3}$ and $F_{5}$. 


\section{Quadrangulating a plane Halin graph}

In deriving the genus distribution of Halin graphs, the critical problem was to invent a new form of decomposition of a plane Halin graph into "atomic" fragments whose genus distributions are known, a new form of amalgamation, and an order of reassembly that reconstructs the Halin graph from the atomic fragments. In this section, we concentrate on the decomposition and the reassembly.

Taking the inscribed tree of a Halin graph as a spanning tree, an edge of a Halin graph is a tree-edge if it lies in the inscribed tree and a cycle-edge if it lies on the outer cycle. A leaf-edge is a tree-edge that is incident at a vertex of the outer cycle. A vertex is called a cycle vertex if it lies on the outer cycle, or an interior tree-vertex otherwise.

We regard the vertices and the edges of the given plane Halin graph as black. We observe that since $H$ is a Halin graph, there is exactly one cycle edge on each polygonal face of the plane embedding. The decomposition is a 4-step process.

Step 1. In each cycle edge of the Halin graph, insert a red midpoint. This is illustrated in Figure 13.

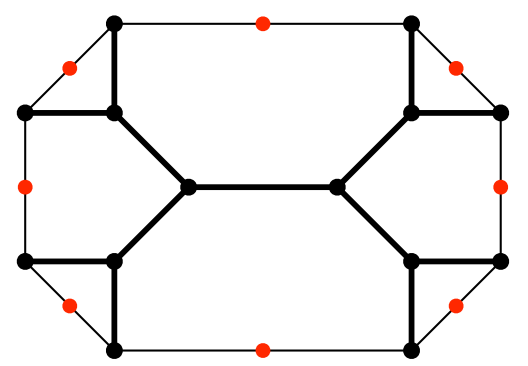

Figure 13: Halin graph plus red midpoints on the exterior cycle.

Step 2. Join each red vertex $v$ to all of the non-leaf vertices on the boundary of the face in whose boundary $v$ lies, as illustrated in Figure 14.

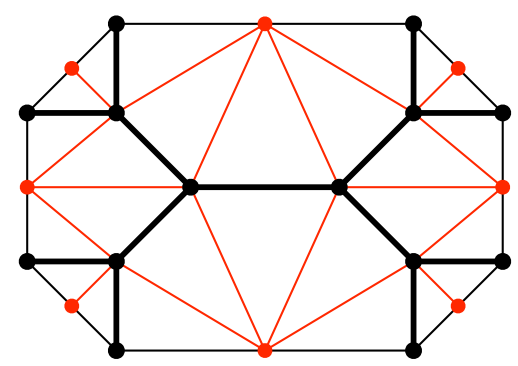

Figure 14: Halin graph plus all of the red edges.

Proposition 3.1. The red and black edges together triangulate the region inside the exterior cycle of a plane Halin graph $G$.

Proof. The black edges create a set of polygons (whose number equals the cycle rank $\beta(G)$ ). Each of these polygons is triangulated by the red edges. 
Proposition 3.2. Every black tree edge lies on two of the triangles formed by Steps 1 and 2 . Proof. Every tree edge lies on two of the polygonal faces of the plane Halin graph (by the Jordan curve theorem). In each of those polygonal faces, it lies on one and only one of the triangles.

Step 3a. For each black tree edge, we pair the two incident triangles into a quadrangle.

Step 3b. We assign (unseen) colors blue, green, and brown to the tree edges, so as to form a proper edge 3-coloring. This is possible because any tree of maximum degree 3 is edge-3-colorable (via greedy algorithm).

Step 3c. We visibly color each quadrangle with the unseen color of the tree edge that bisects it. The coloring of the quadrangles is a proper 3-coloring of the part of the plane inside the exterior cycle of the Halin graph, because of the way it is induced by the proper 3edge-coloring of the tree. (This property will not be used, but it is interesting nonetheless.)

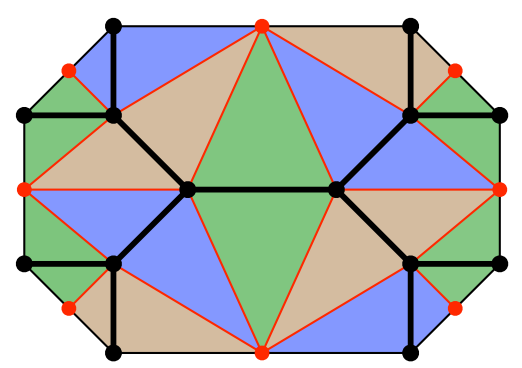

Figure 15: Quadrangulation of a plane Halin graph.

Step 4. Separate the quadrangulated map into quadrangles, and label the interior treevertices.

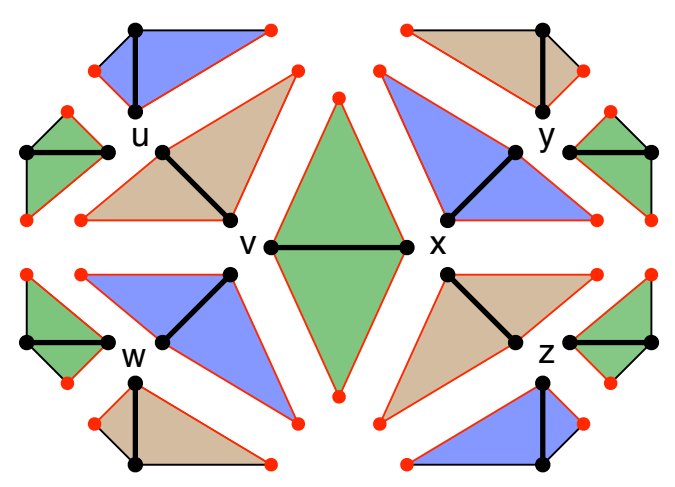

Figure 16: Separated quadrangles of a plane Halin graph. 


\section{Reassembling a Halin graph from its quadrangles: a puzzle}

The success of our method of calculation the genus distribution in the subsequent sections depends on our ability to reassemble the plane Halin graph from its separated quadrangles in a manner consistent with a puzzle now to be described. The genus distributions of the quadrangular fragments is known, and it will be shown that we can calculate the genus distribution of any graph that can be constructed from quadrangular fragments, according to the rules of this puzzle. After giving the rules for this puzzle, we consider the outcome of three attempts at its solution.

\section{Quadrangulation puzzle for a plane cubic Halin graph $H \rightarrow S_{0}$}

1. Each quadrangle $Q$ is regarded as an initial fragment.

2. An $\boldsymbol{R} \boldsymbol{R}$-path on a fragment boundary is a 2-path with two red edges, from a red vertex through a black vertex to another red vertex.

3. Initially, all RR-paths are said to be live.

4. A legal move is initiated by choosing a vertex $v$ such that $v$ is previously unchosen, at least one fragment at $v$ is a quadrangle, and all three RR-paths through $v$ are live RR-paths.

If these three conditions are satisfied, then the three fragments that meet at $v$ are merged into a single (larger) fragment. If there is more than one live RR-path on the boundary of the merged fragment, then all but one of the live RR-paths are deemed to be dead.

5. You LOSE if you run out of legal moves before the map is fully reassembled. This happens whenever there occurs an unmerged vertex $w$ such that either there is a dead RR-path through $w$, or none of the fragments meeting at $w$ is a quadrangle.

6. You WIN the game by reassembling the plane map.

Attempt 1. Start with a merger at $v$. There are three live RR-paths on the boundary of the merged fragment. You LOSE, because RR-paths through two of the unmerged vertices $u, w, x$ become dead.

live RR-path

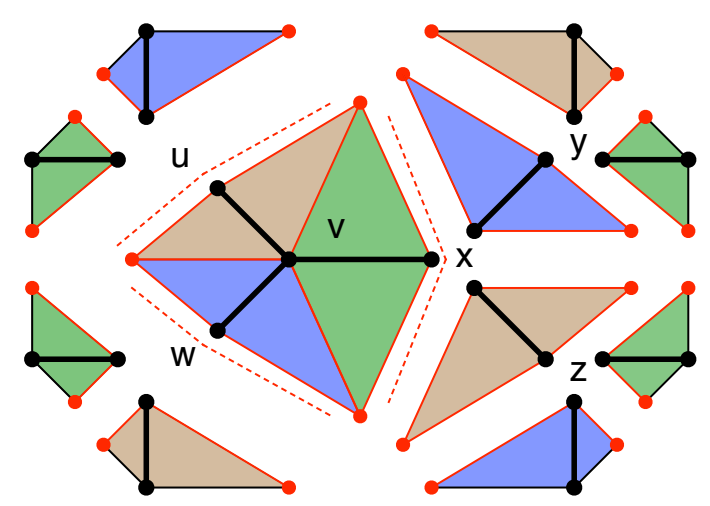

Figure 17: Attempt \#1. 
Attempt 2. First choose $u$ and then choose $v$. There are two live RR-paths on the boundary of the merged fragment. You LOSE, because the RR-path through one of the unmerged vertices $w, x$ becomes dead.

live RR-path

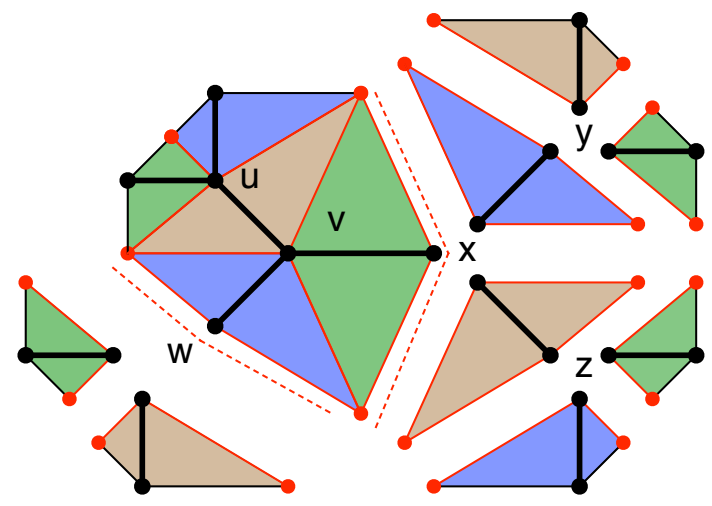

Figure 18: Attempt \#2.

Attempt 3. Start with $u, w, y, z$. You LOSE, since after there is a merger at $v$ or $x$, there will be no quadrangle at the remaining unmerged vertex.

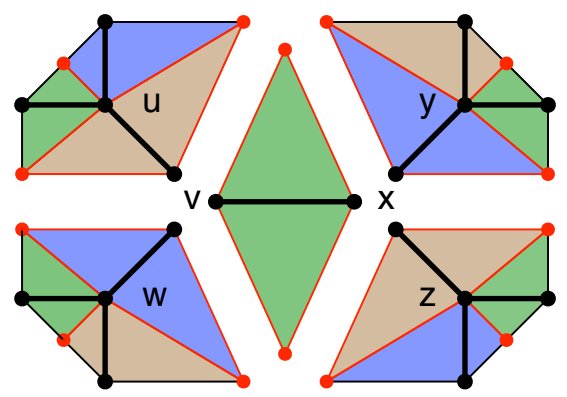

Figure 19: Attempt \#3.

\section{Solution: post-order traversal}

The post-order for the vertices of a plane tree is the order produced when one traces the boundary of the only region and calls out the name of a vertex only the last time it is visited. For the tree in Figure 20, the post-order is $z, y, x, u, v, w$.

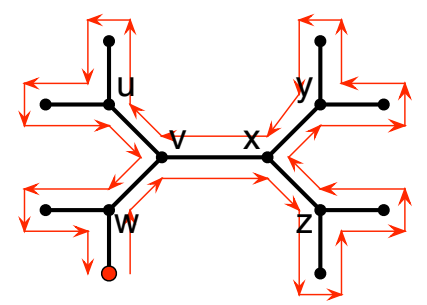

Figure 20: Post-order traversal. 


\section{Solution for the quadrangulation puzzle}

1. As a root for the inscribed tree of the Halin graph, choose any leaf-vertex. (Must be a leaf to win.)

2. Choose vertices in the order in which they occur on a post-order traversal of the tree.

SOLUTION to puzzle in Figure 16: post-order as shown in Figure 20.

$$
\begin{array}{llllll}
z & y & x & u & v & w
\end{array}
$$
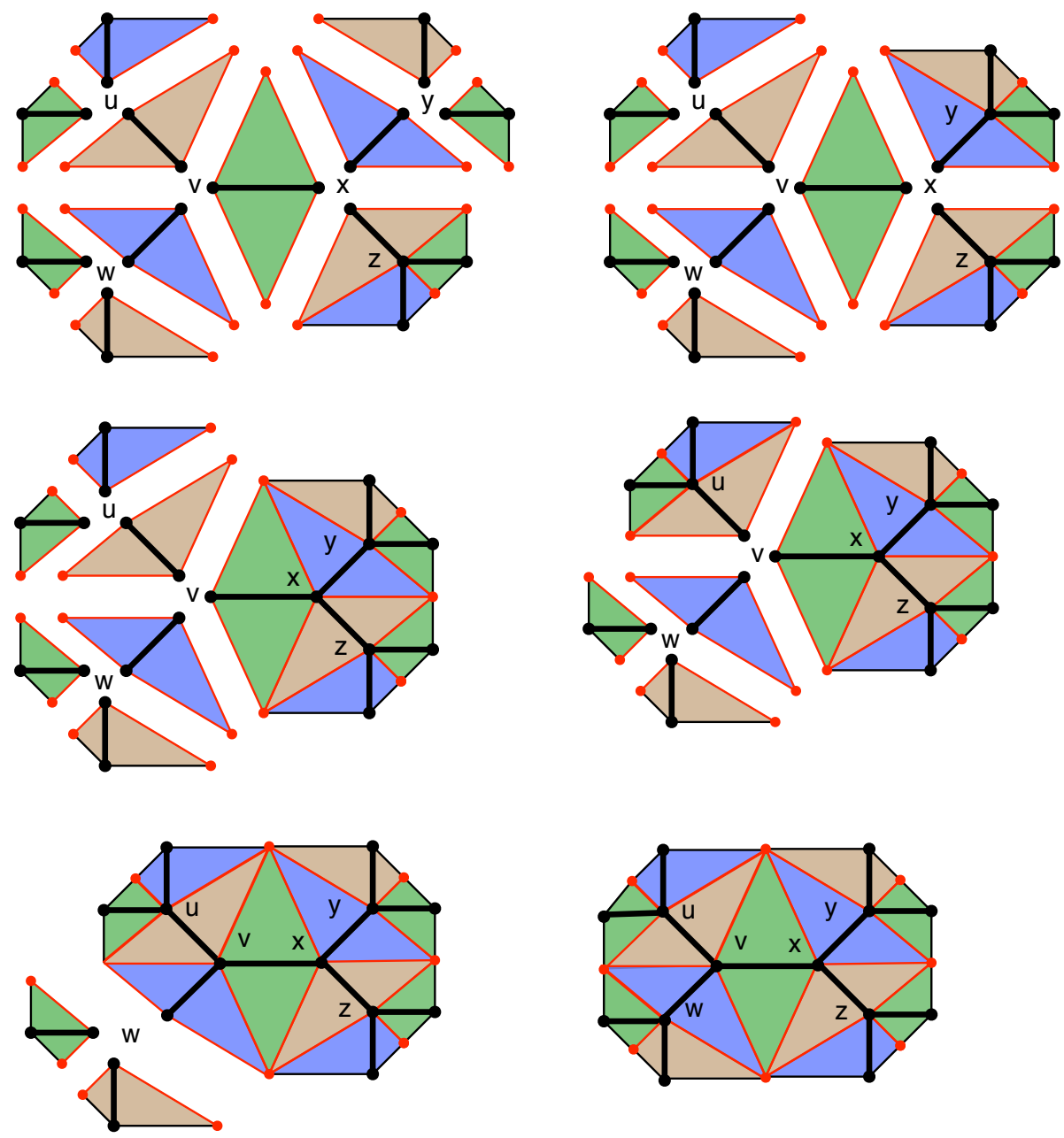

Figure 21: Solving the puzzle with post-order traversal. 
Theorem 3.3. Using the post-order of the interior tree-vertices as the order of merger solves the quadrangle puzzle for any plane cubic Halin graph.

Proof. When the post-order is used, every RR-path through every vertex that follows the vertices of the fragment remains live. It also ensures that there is at least one quadrangle incident on each of those subsequent vertices.

REMARK Quadrangulation and using the post-order solves the generalized puzzle for any Halin graph. The generalized algorithm is not presented only because its details are far lengthier than for the 3-regular case.

\section{Partials and productions for Halin graphs}

When a graph $G$ has one or more of its vertices or edges designated as roots, its genus distribution can be partitioned according to the ways in which face-boundary walks are incident on the roots. The components of such partitions are called partials. A surfaceby-surface inventory of the values of the partials is called a partitioned genus distribution. Such partitioning has been a crucial step in most of the calculations of genus distributions.

Here is a general paradigm for calculating of the genus distribution of the graphs in a given graph family $\mathcal{F}$ by various kinds of graph amalgamation. The tricky part is that all of these requirements must be satisfied in coordination with the others.

- Prescribe a set $\mathcal{A}$ of rooted graphs as atomic fragments and a set $\mathcal{M}$ of merging operations, such that every member of $\mathcal{F}$ can be constructed by iterative application of the merging operations to the atomic fragments. We denote the closure of $\mathcal{A}$ under $\mathcal{M}$ as $\overline{\mathcal{A}}$. Thus, $\mathcal{F} \subseteq \overline{\mathcal{A}}$.

- A procedure is designed to determine, from any graph $G$ in $\overline{\mathcal{A}}$, the sequence of application of operations from $\mathcal{M}$ to atomic fragments and to others constructed earlier in the sequence, by which graph $G$ can be obtained.

- An appropriate set of partials is developed for the rooted graphs in $\overline{\mathcal{A}}$.

- For each operation $\mu \in \mathcal{M}$ there is to be developed a set of rules, called productions, is developed, that prescribe the values of the partials of any graph in $\overline{\mathcal{A}}$ from the values of the partials for the fragments that contribute to its construction under the operation $\mu$.

Example 4.1. For the closed-end ladders and for the other kinds as well, the atomic fragments are doubly edge-rooted cycle graphs. The only operation for closed-end ladders is edge-amalgamation, and the order of application is linear. For the circular ladders and the Möbius ladders, there is an additional operation of self-edge-amalgamation, to be applied last. For the Ringel ladders, the additional operation is edge-addition, to be applied last.

Example 4.2. For the cobblestone walks (see [9]), the atomic fragments are doubly vertexrooted cycle graphs. The only operation is vertex-amalgamation. The order of application is linear. 
Example 4.3. For the 3-regular outerplanar graphs [12], the atomic fragments are doubly edge-rooted cycles. The operations are edge-amalgamation and root-popping on a singly edge-rooted graph. The order of operations is the post-order of a tree. For the 4-regular outerplanar graphs, the atomic fragments are doubly vertex-rooted cycles. The operations are vertex-amalgamation and root-popping. The order of operations is again the post-order of a tree.

\section{Atomic fragments and merging operations for Halin graphs}

The atomic fragments for constructing cubic Halin graphs are the quadrangular fragments obtained as in $\S 3$. We regard them here as doubly vertex-rooted. We denote this set of atomic fragments by $\mathcal{A}_{H}$. The only operation is merging three fragments at an interior vertex of the tree, in such a manner that either there is a surviving RR-path through the vertex of the fragment that is last (among the vertices of the fragment) in the post-order, or the Halin graph is fully reassembled.

\section{Order of mergers for Halin graphs}

The order of mergers of fragments is according to the post-order of the tree.

\section{Partials for cubic Halin graphs}

For a doubly vertex-rooted cubic Halin graph $(G, u, v)$, with the roots $u$ and $v$ inserted at the midpoints of adjacent edges, we split $g_{i}(G)$ into six partials. Here is what they count:

$d d^{\prime}$ Each of the roots $u$ and $v$ lies on two distinct fb-walks. One and only one of these fb-walks traverses both roots.

$d d^{\prime \prime}$ Each of the roots $u$ and $v$ lies on two distinct fb-walks. Both of these fb-walks traverse both roots.

$d s^{\prime}$ Root $u$ lies on two distinct fb-walks. One of these fb-walks traverses root $v$ twice.

$s d^{\prime}$ Root $v$ lies on two distinct fb-walks. One of these fb-walks traverses root $u$ twice.

$s s^{1}$ A single fb-walks traverses roots $u$ and $v$ twice. The occurrences of each root are consecutive.

$s s^{2}$ A single fb-walks traverses roots $u$ and $v$ twice. The occurrences of the two roots alternate.

These configurations are illustrated in Figure 22.
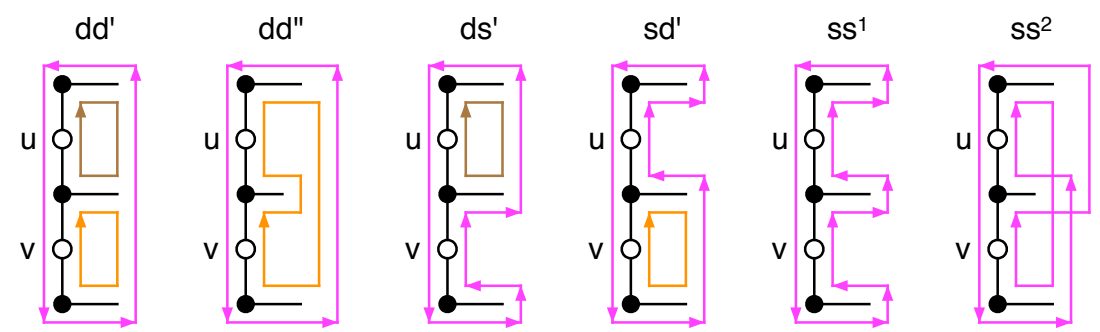

Figure 22: The 6 double-rooted partials for a 3-way pie-merge. 
Proposition 4.1. Let $G$ be any graph that is homeomorphic to a cubic graph, and let its vertex roots $u$ and $v$ be 2-valent endpoints of a pair of edges that are adjacent at a 3-valent vertex. Then the six partials $d d^{\prime}, d d^{\prime \prime}, d s^{\prime}, s d^{\prime}, s s^{1}$, and $s s^{2}$ completely partition the genus distribution of $G$.

Proof. In every embedding of $G$, since $u$ and $v$ lie on a pair of edges that are adjacent at a 3 -valent vertex, there is necessarily an fb-walk on which both of them occur. Thus, if both roots lie on two different fb-walks, $d d^{\prime}$ and $d d^{\prime \prime}$ are the only possibilities. If one lies on two different fb-walks and the other on only one fb-walk, then $d s^{\prime}$ and $s d^{\prime}$ are the only possibilities. If both roots occur twice on the same fb-walk, then either $\left(s s^{1}\right)$ the occurrences of each root are consecutive, or $\left(s s^{2}\right)$ they alternate.

\section{Productions for cubic Halin graphs}

For cubic Halin graphs, we merge three graphs at a time, exactly as for the puzzle, so that one of them is a quadrangle $Q=K_{4}-e$, with its two roots inserted at the midpoints of the two quadrangle boundary edges that meet at the vertex to be merged. Envisioning this configuration at a small pie cut into three slices, we call the a 3 -way $\pi$-merge. It is illustrated in Figure 23.

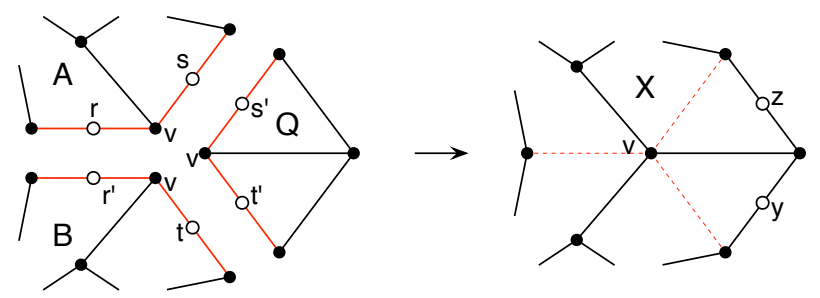

Figure 23: A 3-way $\pi$-merge $\left((A, r, s),\left(B, t, r^{\prime}\right),\left(Q, s^{\prime}, t^{\prime}\right)\right) \rightarrow(X, y, z)$ at vertex $v$.

Proposition 4.2. In a 3-way $\pi$-merge $(A, B, Q) \rightarrow X$ at vertex $v$, each rotation system $\rho$ for $X$ is consistent with exactly two rotation systems for fragment $A$ and exactly two for fragment $B$.

Proof. If rotation system $\rho$ is consistent with a given rotation system $\rho_{A}$ of fragment $A$, then it is also consistent with the rotation system of $A$ obtained from $\rho_{A}$ by reversing the rotation at $v$. A similar observation holds for fragment $B$.

Suppose that $p^{1}, p^{2}, \ldots, p^{s}$ is a set of partials or subpartials for a genus distribution. A production for a given surgical operation that transforms either a graph embedding $X \rightarrow S_{i}$ (or a tuple of graph embeddings) into a set of graph embeddings of the graph $Y$ is a rule of this form:

$$
p_{i}^{j}(X) \longrightarrow c_{1} p_{f_{1}^{j}(i)}^{1}(Y)+\cdots+c_{t} p_{f_{s}^{j}(i)}^{s}(Y)
$$

The left side is called the antecedent, and the right side is called the consequent. The meaning is that the operation transforms a single embedding of graph $X$ of type $p^{j}$ on the surface $S_{i}$ into a set of embeddings of the graph $Y$, of which $c_{k}$ are of type $p^{k}$ on the surface $S_{f_{k}^{j}(i)}$, for each $i, j$, and $k$. A drawing is usually used as an aid in deriving the production and in proving its correctness. The names of the graphs and their roots can be suppressed when there is in context no ambiguity. 
Example 4.4. One of the productions for the $\pi$-merge of doubly vertex-rooted graphs $(A, r, s),\left(B, t, r^{\prime}\right)$ and $\left(Q, s^{\prime}, t^{\prime}\right)$ into $(X, y, z)$ is

$$
d d_{i}^{\prime}(A, r, s) * d d_{j}^{\prime \prime}\left(B, t, r^{\prime}\right) \longrightarrow 2 d d_{i+j}^{\prime}(X, y, z)+2 s s_{i+j+1}^{2}(X, y, z)
$$

It means that a type- $d d^{\prime}$ embedding $(A, r, s) \rightarrow S_{i}$ and a type- $d d^{\prime \prime}$ embedding $\left(B, t, r^{\prime}\right) \rightarrow$ $S_{j}$ combine into two type- $d d^{\prime}$ embeddings $(X, y, z) \rightarrow S_{i+j}$ and two type-ss $s^{2}$ embeddings $(X, y, z) \rightarrow S_{i+j+1}$. The relevant drawing is shown in Figure 24 .
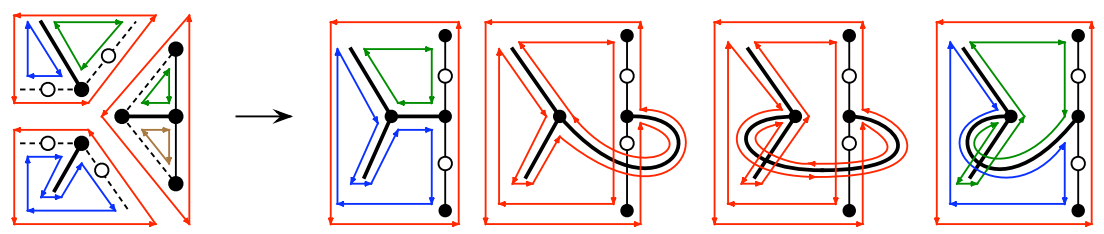

Figure 24: Prod \#2: $d d_{i}^{\prime} * d d_{j}^{\prime \prime} \longrightarrow 2 d d_{i+j}^{\prime}+2 s s_{i+j+1}^{2}$.

Theorem 4.3. The following 36 productions are a complete set of rules for calculating the genus distribution of the graph that results from a $\pi$-merge of three graphs in $\overline{\mathcal{A}_{H}}$.

1. $d d_{i}^{\prime} * d d_{j}^{\prime} \longrightarrow d d_{i+j}^{\prime}+2 d d_{i+j+1}^{\prime \prime}+s s_{i+j+1}^{2}$.

2. $d d_{i}^{\prime} * d d_{j}^{\prime \prime} \longrightarrow 2 d d_{i+j}^{\prime}+2 s s_{i+j+1}^{2}$.

3. $d d_{i}^{\prime} * d s_{j}^{\prime} \longrightarrow 2 d d_{i+j}^{\prime}+2 s s_{i+j+1}^{2}$.

4. $d d_{i}^{\prime} * s d_{j}^{\prime} \longrightarrow 2 s d_{i+j}^{\prime}+2 s s_{i+j+1}^{1}$.

5. $d d_{i}^{\prime} * s s_{j}^{1} \longrightarrow 4 s d_{i+j}^{\prime}$.

6. $d d_{i}^{\prime} * s s_{j}^{2} \longrightarrow 2 d s_{i+j}^{\prime}+2 s d_{i+j}^{\prime}$.

7. $d d_{i}^{\prime \prime} * d d_{j}^{\prime} \longrightarrow 2 d d_{i+j}^{\prime}+2 s s_{i+j+1}^{2}$.

8. $d d_{i}^{\prime \prime} * d d_{j}^{\prime \prime} \longrightarrow 4 d d_{i+j}^{\prime \prime}$.

9. $d d_{i}^{\prime \prime} * d s_{j}^{\prime} \longrightarrow 4 d s_{i+j}^{\prime}$.

10. $d d_{i}^{\prime \prime} * s d_{j}^{\prime} \longrightarrow 4 s d_{i+j}^{\prime}$.

11. $d d_{i}^{\prime \prime} * s s_{j}^{1} \longrightarrow 4 s s_{i+j}^{1}$.

12. $d d_{i}^{\prime \prime} * s s_{j}^{2} \longrightarrow 2 d d_{i+j-1}^{\prime}+2 s s_{i+j}^{2}$.

13. $d s_{i}^{\prime} * d d_{j}^{\prime} \longrightarrow 2 d s_{i+j}^{\prime}+2 s s_{i+j+1}^{1}$.

14. $d s_{i}^{\prime} * d d_{j}^{\prime \prime} \longrightarrow 4 d s_{i+j}^{\prime}$.

15. $d s_{i}^{\prime} * d s_{j}^{\prime} \longrightarrow 4 d s_{i+j}^{\prime}$.

16. $d s_{i}^{\prime} * s d_{j}^{\prime} \longrightarrow 4 s s_{i+j}^{1}$.

17. $d s_{i}^{\prime} * s s_{j}^{1} \longrightarrow 4 s s_{i+j}^{1}$.

18. $d s_{i}^{\prime} * s s_{j}^{2} \longrightarrow 2 d s_{i+j-1}^{\prime}+2 s s_{i+j}^{1}$.

19. $s d_{i}^{\prime} * d d_{j}^{\prime} \longrightarrow 2 d d_{i+j}^{\prime}+2 s s_{i+j+1}^{2}$. 
20. $s d_{i}^{\prime} * d d_{j}^{\prime \prime} \longrightarrow 4 s d_{i+j}^{\prime}$.

21. $s d_{i}^{\prime} * d s_{j}^{\prime} \longrightarrow 2 d d_{i+j-1}^{\prime}+2 s s_{i+j}^{2}$.

22. $s d_{i}^{\prime} * s d_{j}^{\prime} \longrightarrow 4 s d_{i+j}^{\prime}$.

23. $s d_{i}^{\prime} * s s_{j}^{1} \longrightarrow 4 s d_{i+j-1}^{\prime}$.

24. $s d_{i}^{\prime} * s s_{j}^{2} \longrightarrow 2 d d_{i+j-1}^{\prime}+2 s s_{i+j}^{2}$.

25. $s s_{i}^{1} * d d_{j}^{\prime} \longrightarrow 4 d s_{i+j}^{\prime}$.

26. $s s_{i}^{1} * d d_{j}^{\prime \prime} \longrightarrow 4 s s_{i+j}^{1}$.

27. $s s_{i}^{1} * d s_{j}^{\prime} \longrightarrow 4 d s_{i+j-1}^{\prime}$.

28. $s s_{i}^{1} * s d_{j}^{\prime} \longrightarrow 4 s s_{i+j}^{1}$.

29. $s s_{i}^{1} * s s_{j}^{1} \longrightarrow 4 s s_{i+j-1}^{1}$.

30. $s s_{i}^{1} * s s_{j}^{2} \longrightarrow 4 d s_{i+j-1}^{\prime}$.

31. $s s_{i}^{2} * d d_{j}^{\prime} \longrightarrow 2 d s_{i+j}^{\prime}+2 s d_{i+j}^{\prime}$.

32. $s s_{i}^{2} * d d_{j}^{\prime \prime} \longrightarrow 2 d d_{i+j-1}^{\prime}+2 s s_{i+j}^{2}$.

33. $s s_{i}^{2} * d s_{j}^{\prime} \longrightarrow 2 d d_{i+j-1}^{\prime}+2 s s_{i+j}^{2}$.

34. $s s_{i}^{2} * s d_{j}^{\prime} \longrightarrow 2 s d_{i+j-1}^{\prime}+2 s s_{i+j}^{1}$.

35. $s s_{i}^{2} * s s_{j}^{1} \longrightarrow 4 s d_{i+j-1}^{\prime}$.

36. $s s_{i}^{2} * s s_{j}^{2} \longrightarrow 2 d d_{i+j-1}^{\prime \prime}+d d_{i+j-2}^{\prime}+s s_{i+j-1}^{2}$.

Proof. The correctness of each of these productions is a matter of recombining the strands as prescribed by the $\pi$-merge. The 36 figures corresponding to these productions are given by [14].

\section{Computational Complexity}

Theorem 4.4. For $\left|V_{A}\right|=k$ and $\left|V_{B}\right|=m$, there is an $O(k m)$-time algorithm for calculating the partitioned genus distribution of the resulting graph $X$ of a 3-way pie-merge $(A, B, Q) \rightarrow X$ of graphs whose maximum degree is 3.

Proof. The number of non-zero partials of a cubic graph $G$ with $p$ vertices is in $O(p)$, since the maximum genus cannot exceed $\beta(G) / 2$. For each non-zero-valued partial of $A$ and each non-zero-valued partial of $B$, only one production is applied, and the time for the application of a single production is in $O(1)$.

Corollary 4.5. The post-order traversal using the 36 productions corresponding to the six partials yields an $O\left(n^{2}\right)$ algorithm for the genus distribution of a cubic Halin graph with $n$ vertices.

Proof. Let $H$ have quadrangular fragments $Q_{1}, \ldots, Q_{f}$ of respective cardinalities $q_{1}, \ldots$, $q_{f}$. The number of non-zero-valued partials in the $\pi$-merge of a $k$-vertex fragment $A$ with an $m$-vertex fragment $B$ and a quadrangular fragment $Q_{i}$ is at most a constant multiple of $k+m$. Since each pair of initial quadrangular fragments is merged only once during 
the reassembly of the Halin graph, it follows that the number of steps is at most a constant multiple of the sum

$$
\sum_{i \neq j} q_{i} q_{j}
$$

where $q_{i}$ is the number of non-zero partials of the quadrangular fragment $Q_{i}$. However,

$$
\sum_{i \neq j} q_{i} q_{j}<\left(q_{1}+q_{2}+\cdots+q_{f}\right)^{2}
$$

The conclusion follows.

\section{Sample Calculation}

In this section, we show the work needed to calculate the genus distribution of the Halin graph of Figure 1.

\section{Merger at $z$}

$\operatorname{Graph} \mathbf{A}\left(K_{4}-e\right)$ :

\begin{tabular}{||r|r|r|r|r|r|r||r||}
\hline \hline $\mathrm{i}$ & $d d_{i}^{\prime}$ & $d d_{i}^{\prime \prime}$ & $d s_{i}^{\prime}$ & $s d_{i}^{\prime}$ & $s s_{i}^{1}$ & $s s_{i}^{2}$ & $g_{i}$ \\
\hline 0 & 2 & 0 & 0 & 0 & 0 & 0 & 2 \\
1 & 0 & 0 & 0 & 0 & 0 & 2 & 2 \\
\hline \hline
\end{tabular}

Graph B $\left(K_{4}-e\right)$ :

\begin{tabular}{||r|r|r|r|r|r|r||r||}
\hline \hline $\mathrm{i}$ & $d d_{i}^{\prime}$ & $d d_{i}^{\prime \prime}$ & $d s_{i}^{\prime}$ & $s d_{i}^{\prime}$ & $s s_{i}^{1}$ & $s s_{i}^{2}$ & $g_{i}$ \\
\hline 0 & 2 & 0 & 0 & 0 & 0 & 0 & 2 \\
1 & 0 & 0 & 0 & 0 & 0 & 2 & 2 \\
\hline \hline
\end{tabular}

Merged Graph $K_{4}$ : Use Productions 1, 6, 31, and 36.

\begin{tabular}{||r|r|r|r|r|r|r||r||}
\hline \hline $\mathrm{i}$ & $d d_{i}^{\prime}$ & $d d_{i}^{\prime \prime}$ & $d s_{i}^{\prime}$ & $s d_{i}^{\prime}$ & $s s_{i}^{1}$ & $s s_{i}^{2}$ & $g_{i}$ \\
\hline 0 & 2 & 0 & 0 & 0 & 0 & 0 & 2 \\
1 & 0 & 4 & 4 & 4 & 0 & 2 & 14 \\
\hline \hline
\end{tabular}

\section{Merger at $y$}

Merged Graph $K_{4}$ : Just like the merger at $z$.

\begin{tabular}{||r|r|r|r|r|r|r||r||}
\hline \hline $\mathrm{i}$ & $d d_{i}^{\prime}$ & $d d_{i}^{\prime \prime}$ & $d s_{i}^{\prime}$ & $s d_{i}^{\prime}$ & $s s_{i}^{1}$ & $s s_{i}^{2}$ & $g_{i}$ \\
\hline 0 & 2 & 0 & 0 & 0 & 0 & 0 & 2 \\
1 & 0 & 4 & 4 & 4 & 0 & 2 & 14 \\
\hline \hline
\end{tabular}

\section{Merger at $x$}

\section{Graph A (result from merger at $z$ ):}

\begin{tabular}{||r|r|r|r|r|r|r||r||}
\hline \hline $\mathrm{i}$ & $d d_{i}^{\prime}$ & $d d_{i}^{\prime \prime}$ & $d s_{i}^{\prime}$ & $s d_{i}^{\prime}$ & $s s_{i}^{1}$ & $s s_{i}^{2}$ & $g_{i}$ \\
\hline 0 & 2 & 0 & 0 & 0 & 0 & 0 & 2 \\
1 & 0 & 4 & 4 & 4 & 0 & 2 & 14 \\
\hline \hline
\end{tabular}


Graph B (result from merger at $y$ ):

\begin{tabular}{||r|r|r|r|r|r|r||r||}
\hline \hline $\mathrm{i}$ & $d d_{i}^{\prime}$ & $d d_{i}^{\prime \prime}$ & $d s_{i}^{\prime}$ & $s d_{i}^{\prime}$ & $s s_{i}^{1}$ & $s s_{i}^{2}$ & $g_{i}$ \\
\hline 0 & 2 & 0 & 0 & 0 & 0 & 0 & 2 \\
1 & 0 & 4 & 4 & 4 & 0 & 2 & 14 \\
\hline \hline
\end{tabular}

Merged Graph: Use 25 productions (all those without the partial $s s^{1}$ ).

\begin{tabular}{||r|r|r|r|r|r|r|r||}
\hline \hline $\mathrm{i}$ & $d d_{i}^{\prime}$ & $d d_{i}^{\prime \prime}$ & $d s_{i}^{\prime}$ & $s d_{i}^{\prime}$ & $s s_{i}^{1}$ & $s s_{i}^{2}$ & $g_{i}$ \\
\hline 0 & 2 & 0 & 0 & 0 & 0 & 0 & 2 \\
1 & 40 & 4 & 12 & 12 & 0 & 2 & 70 \\
2 & 0 & 16 & 48 & 48 & 32 & 40 & 184 \\
\hline \hline
\end{tabular}

Merger at $u$

Merged Graph: $K_{4}$ : Just like the merger at $z$.

\begin{tabular}{||r|r|r|r|r|r|r||r||}
\hline \hline $\mathrm{i}$ & $d d_{i}^{\prime}$ & $d d_{i}^{\prime \prime}$ & $d s_{i}^{\prime}$ & $s d_{i}^{\prime}$ & $s s_{i}^{1}$ & $s s_{i}^{2}$ & $g_{i}$ \\
\hline 0 & 2 & 0 & 0 & 0 & 0 & 0 & 2 \\
1 & 0 & 4 & 4 & 4 & 0 & 2 & 14 \\
\hline \hline
\end{tabular}

Merger at $v$

Graph A (result from merger at $x)$ :

\begin{tabular}{||r|r|r|r|r|r|r|r||}
\hline \hline $\mathrm{i}$ & $d d_{i}^{\prime}$ & $d d_{i}^{\prime \prime}$ & $d s_{i}^{\prime}$ & $s d_{i}^{\prime}$ & $s s_{i}^{1}$ & $s s_{i}^{2}$ & $g_{i}$ \\
\hline 0 & 2 & 0 & 0 & 0 & 0 & 0 & 2 \\
1 & 40 & 4 & 12 & 12 & 0 & 2 & 70 \\
2 & 0 & 16 & 48 & 48 & 32 & 40 & 184 \\
\hline \hline
\end{tabular}

Graph B (result from merger at $u$ ):

\begin{tabular}{||r|r|r|r|r|r|r||r||}
\hline \hline $\mathrm{i}$ & $d d_{i}^{\prime}$ & $d d_{i}^{\prime \prime}$ & $d s_{i}^{\prime}$ & $s d_{i}^{\prime}$ & $s s_{i}^{1}$ & $s s_{i}^{2}$ & $g_{i}$ \\
\hline 0 & 2 & 0 & 0 & 0 & 0 & 0 & 2 \\
1 & 0 & 4 & 4 & 4 & 0 & 2 & 14 \\
\hline \hline
\end{tabular}

Merged Graph: Use 30 productions.

\begin{tabular}{||r|r|r|r|r|r|r||r||}
\hline \hline $\mathrm{i}$ & $d d_{i}^{\prime}$ & $d d_{i}^{\prime \prime}$ & $d s_{i}^{\prime}$ & $s d_{i}^{\prime}$ & $s s_{i}^{1}$ & $s s_{i}^{2}$ & $g_{i}$ \\
\hline 0 & 2 & 0 & 0 & 0 & 0 & 0 & 2 \\
1 & 112 & 4 & 28 & 12 & 0 & 2 & 158 \\
2 & 544 & 96 & 544 & 352 & 80 & 112 & 1728 \\
3 & 0 & 64 & 448 & 448 & 704 & 544 & 2208 \\
\hline \hline
\end{tabular}




\section{Merger at $w$}

Graph A (result from merger at $v$ ):

\begin{tabular}{||r|r|r|r|r|r|r||r||}
\hline \hline $\mathrm{i}$ & $d d_{i}^{\prime}$ & $d d_{i}^{\prime \prime}$ & $d s_{i}^{\prime}$ & $s d_{i}^{\prime}$ & $s s_{i}^{1}$ & $s s_{i}^{2}$ & $g_{i}$ \\
\hline 0 & 2 & 0 & 0 & 0 & 0 & 0 & 2 \\
1 & 112 & 4 & 28 & 12 & 0 & 2 & 158 \\
2 & 544 & 96 & 544 & 352 & 80 & 112 & 1728 \\
3 & 0 & 64 & 448 & 448 & 704 & 544 & 2208 \\
\hline \hline
\end{tabular}

Graph B $\left(K_{4}-e\right)$ :

\begin{tabular}{||r|r|r|r|r|r|r||r||}
\hline \hline $\mathrm{i}$ & $d d_{i}^{\prime}$ & $d d_{i}^{\prime \prime}$ & $d s_{i}^{\prime}$ & $s d_{i}^{\prime}$ & $s s_{i}^{1}$ & $s s_{i}^{2}$ & $g_{i}$ \\
\hline 0 & 2 & 0 & 0 & 0 & 0 & 0 & 2 \\
1 & 0 & 0 & 0 & 0 & 0 & 2 & 2 \\
\hline \hline
\end{tabular}

\section{Merged Graph: final result}

\begin{tabular}{||r|r|r|r|r|r|r||r||}
\hline \hline $\mathrm{i}$ & $d d_{i}^{\prime}$ & $d d_{i}^{\prime \prime}$ & $d s_{i}^{\prime}$ & $s d_{i}^{\prime}$ & $s s_{i}^{1}$ & $s s_{i}^{2}$ & $g_{i}$ \\
\hline 0 & 2 & 0 & 0 & 0 & 0 & 0 & 2 \\
1 & 144 & 4 & 60 & 4 & 0 & 2 & 214 \\
2 & 1440 & 224 & 1632 & 224 & 56 & 144 & 3720 \\
3 & 1024 & 1088 & 4800 & 1088 & 1088 & 1440 & 10528 \\
4 & 0 & 0 & 0 & 0 & 896 & 1024 & 1920 \\
\hline \hline
\end{tabular}

\section{Conclusions}

We have demonstrated the usefulness of the paradigm given at the beginning of $\S 4$ in deriving a practical algorithm for the genus distribution of cubic Halin graphs. To be practical, in the sense intended here, the number of partials needed should be relatively small.

To calculate the genus distribution of a family of graphs, under this paradigm, one first designs a recursive specification of that family, that is, a finite set of base graphs and a finite set of operations whose iterative application can construct any graph in the family. One then derives a set of production rules for obtaining the partitioned genus distribution of the result of the applying any operation from the partitioned genus distributions of the operands.

There are problems whose general solution seems to require exponentially large effort, but which can be solved in polynomial-time for cases in which something is bounded. A familiar result in topological graph theory is that whereas Thomassen [36] proved that determining the minimum genus of a graph is NP-hard, Mohar [27] proved that for every possible orientable surface $S_{i}$, there is a linear-time algorithm to decide whether a given graph is embeddable in that surface.

Shortly after the presentation of this paper, the author derived, for any fixed treewidth and maximum degree, a quadratic-time algorithm [15] to calculate the genus distribution of any graph conforming to those bounds. This algorithm is less than practical, since the numbers of partials and productions increase exponentially with the treewidth and the maximum degree. 


\section{References}

[1] L. W. Beineke, R. J. Wilson, J. L. Gross and T. W. Tucker, Editors, Topics in Topological Graph Theory, Cambridge Univ. Press, 2009.

[2] H. L. Bodlaender, A partial $k$-arboretum of graphs with bounded treewidth, Theoretical Comp. Sci. 209 (1998), 1-45.

[3] C. P. Bonnington and C. H. C. Little, The Foundations of Topological Graph Theory, Springer, 1995.

[4] Y. Chen, Lower bounds for the average genus of a CF-graph, Electronic J. Combin. 17 (2010), \#R150.

[5] Y Chen, J. L. Gross and T. Mansour, Genus distributions of star-ladders, Discrete Math. (2012), to appear.

[6] Y. Chen, T. Mansour and Q. Zou, Embedding distributions of generalized fan graphs, Canad. Math. Bull. (2011), online 31aug2011.

[7] J. Chen and J. L. Gross, Limit points for average genus (I): 3-connected and 2-connected simplicial graphs, J. Combin. Theory (B) 55 (1992), 83-103.

[8] M. Furst, J. L. Gross and L. A. McGeoch, Finding a maximum-genus imbedding, J. ACM 35 (1988), 523-534.

[9] M. L. Furst, J. L. Gross and R. Statman, Genus distribution for two classes of graphs, J. Combin. Theory (B) 46 (1989), 22-36.

[10] J. L. Gross, Distribution of embeddings, in: L. W. Beineke, R. J. Wilson, J. L. Gross and T. W. Tucker (eds.), Chapter 3 of Topics in Topological Graph Theory, Cambridge Univ. Press, 2009.

[11] J. L. Gross, Genus distribution of graphs under surgery: adding edges and splitting vertices, New York J. Math. 16 (2010), 161-178.

[12] J. L. Gross, Genus distribution of graph amalgamations: Self-pasting at root-vertices, Australasian J. Combin. 49 (2011), 19-38.

[13] J. L. Gross, Genus distributions of cubic outerplanar graphs, J. of Graph Algorithms and Applications 15 (2011), 295-316.

[14] J. L. Gross, Productions for 3-way $\pi$-merges, http://www.cs.columbia.edu/ gross/supplementary.html.

[15] J. L. Gross, Embeddings of graphs of fixed treewidth and bounded degree, Abstract 1077-051655, Boston Meeting of the Amer. Math. Soc. (Jan. 2012).

[16] J. L. Gross and M. L. Furst, Hierarchy for imbedding-distribution invariants of a graph, J. Graph Theory 11 (1987), 205-220.

[17] J. L. Gross, I. F. Khan and M. I. Poshni, Genus distribution of graph amalgamations: pasting at root-vertices, Ars Combinatoria 94 (2010), 33-53.

[18] J. L. Gross, D. P. Robbins and T. W. Tucker, Genus distributions for bouquets of circles, J. Combin. Theory (B) 47 (1989), 292-306.

[19] J. L. Gross and T. W. Tucker, Topological Graph Theory, Dover, 2001; (original edn. Wiley, 1987).

[20] R. Halin, Über simpliziale Zerfällungen beliebiger (endlicher oder unendlicher) Graphen, Mathematische Annalen 156 (1964), 216-225.

[21] D. M. Jackson, Counting cycles in permutations by group characters, with an application to a topological problem, Trans. Amer. Math. Soc. 299 (1987), 785-801. 
[22] I. F. Khan, M. I. Poshni and J. L. Gross, Genus distribution of graph amalgamations at roots of higher degree, Ars Math. Contemp. 3 (2010), 121-138.

[23] I. F. Khan, M. I. Poshni, and J. L. Gross, Genus distribution of $P_{3} \times P_{n}$, Discrete Math. (2012), to appear.

[24] J. H. Kwak and J. Lee, Genus polynomials of dipoles, Kyungpook Math. J. 33 (1993), 115-125.

[25] J. H. Kwak and J. Lee, Enumeration of graph embeddings, Discrete Math. 135 (1994), 129151.

[26] L. A. McGeoch, Algorithms for two graph problems: computing maximum-genus imbedding and the two-server problem, $\mathrm{PhD}$ thesis, Carnegie-Mellon University, 1987.

[27] B. Mohar, A linear time algorithm for embedding graphs in an arbitrary surface, SIAM J. Discrete Math. 12 (1999), 6-26.

[28] B. Mohar and C. Thomassen, Graphs on Surfaces, Johns Hopkins Press, 2001.

[29] M. I. Poshni, I. F. Khan and J. L. Gross, Genus distribution of edge-amalgamations, Ars Math. Contemporanea 3 (2010), 69-86.

[30] M. I. Poshni, I. F. Khan and J. L. Gross, Genus distribution of 4-regular outerplanar graphs, Electronic J. Combin. 18 (2011), \#P212.

[31] M. I. Poshni, I. F. Khan and J. L. Gross, Genus distribution of graphs under self-edgeamalgamations, Ars Math. Contemp. 5 (2012), 127-148.

[32] R. G. Rieper, The enumeration of graph embeddings, Ph.D. thesis, Western Michigan University, 1990.

[33] S. Stahl, Region distributions of graph embeddings and Stirling numbers, Discrete Math. 82 (1990), 57-78.

[34] S. Stahl, Region distributions of some small diameter graphs, Discrete Math. 89 (1991), 281299.

[35] E. H. Tesar, Genus distribution of Ringel ladders, Discrete Math 216 (2000), 235-252.

[36] C. Thomassen, The graph genus problem is NP-complete, J. Algorithms 10 (1989), 568-576.

[37] A. T. White, Graphs of Groups on Surfaces, North-Holland, 2001. 